\title{
Les superantigènes bactériens : agents pathogènes ou outils thérapeutiques
}

Les entérotoxines, qui provoquent l'empoisonnement alimentaire causé par Staphylococcus aureus, sont des superantigènes. Leurs propriétés au sein du système immunitaire en font des molécules potentiellement dévastatrices dont le rôle dans le développement des maladies auto-immunitaires ne serait pas négligeable. Il semble possible de détourner ces effets néfastes pour éventuellement tirer parti du caractère immunomodulateur des superantigènes modifiés. On disposerait alors d'outils thérapeutiques susceptibles de traiter de nombreuses pathologies.

\section{Jacques Thibodeau Gilbert Croteau Nathalie Labrecque Rafick-Pierre Sékaly}

a plupart des bactéries qui colonisent l'organisme humain sont inoffensives; des barrière naturelles, comme les cellules épithéliales ou le système immunitaire, les empêchent aussitôt de proliférer. Ces bactéries deviennent pathogènes lorsque le système immunitaire s'affaiblit, lorsqu'elles traversent les obstacles physiques comme la peau, ou encore lorsqu'elles synthétisent de nouvelles substances extracellulaires associées à des mécanismes de toxicité. Certaines de ces exoprotéines ont une activité catalytique leur permettant par exemple de modifier des molécules à la surface des cellules de l'hôte et d'augmenter ainsi leur pouvoir d'invasion [1]. Cependant, d'autres sont des superantigènes : elles déclenchent dans le système immunitaire une cascade complexe de réactions qui risquent d'aboutir au choc toxique [2]. Elles stimulent in vitro et in vivo la prolifération et la différenciation d'une très large proportion des cellules $\mathrm{T}$ (10-40\%), contrairement aux antigènes conventionnels qui stimulent moins de 0,01\% des lymphocytes. Ces molécules, efficaces à des concentrations extrêmement faibles $\left(10^{-9} \mathrm{M}\right)$, font partie des mitogènes les plus puissants des cellules T. Les mécanismes de leur toxicité impliquent 1) une prolifération lymphocytaire accompagnée de la synthèse de lymphokines (interleukines-1 et -2, cachectines), 2) le relargage de leucotriènes produites par les cellules mastocytaires [2, 3]. Paradoxalement cette stimulation massive mais incontrôlée du système immunitaire entraînerait une immunosuppression dont la bactérie tire avantage. Plusicurs molécules bactériennes dotées de propriétés superantigéniques ont été décrites (Tableau I); les plus étudiées toutefois sont les entérotoxines produites par Staphylococcus aureus. Nous nologie, IRCM, département de microbi Montréal. 
examinons principalement ici les propriétés de ces toxines. Nous analysons en détail leur structure, ainsi que leurs interactions avec différents ligands des cellules hôtes.

\section{Propriétés physico-chimiques des entérotoxines}

Des colonies de bactéries de la famille Staphyloccocus aureus occupent la peau de façon naturelle. Différentes souches produisent des exotoxines dont on connaît plusieurs sérotypes (A, B, C1, C2, C3, D, E). L'ingestion d'aliments mal conservés et contaminés par une souche de $S$. aureus, courtcircuite les barrières naturelles et expose l'hôte aux effets de ces exotoxines. Ces molécules sont responsables de près de $25 \%$ des empoisonnements alimentaires et des dérangements entériques [4]. Les entérotoxines sont relativement résistantes à la protéolyse et pratiquement insensibles à la chaleur. Elles ont une masse moléculaire relative variant de 26000 à 30000 . Elles peuvent partager jusqu'à $90 \%$ de similitude au niveau de leur structure primaire comme c'est le cas entre SEA et SEE [2]. Elles comportent une faible proportion d'hélices $\alpha$, mais plusieurs feuillets $\beta$ [5]. La présence de résidus de cystéines permet la formation d'un pont disulfure qui prend l'aspect d'une courte boucle en position centrale dans plusieurs de ces molécules.

Dans les années 80 , une entérotoxine de $S$. aureus ne possédant pas de pont disulfure a été identifiée. Il s'agit de la toxine du syndrome du choc toxique (TSST-1). Elle diffère beaucoup des autres entérotoxines avec lesquelles elle ne partage guère que $45 \%$ de similitude. Cette toxine ne provoque pas systématiquement d'effets entérotoxiques chez le singe; en revanche, elle affecte de façon significative l'organisme humain ; par exemple, elle est associée chez la femme au choc toxique que causent certains tampons hygiéniques. Les gènes codant pour ces molécules sont chromosomiques ou plasmidiques. Certains (SEA et SPEA) sont localisés sur des bactériophages intégrés dans le génome bactérien et sont donc " mobiles" [1].

\section{Propriétés biologiques des entérotoxines}

On est loin d'avoir prouvé que les entérotoxines absorbées par un humain passent dans la circulation. Cependant, des singes auxquels on a fait ingérer des entérotoxines ont produit des anticorps protecteurs. La quantité de toxines retrouvée dans les fluides biologiques est à peine détectable ; ces toxines seraient rapidement ćliminées par l'organisme. Ainsi, lorsque des singes subissent des injections de SEB, $60 \%$ de la dose disparaît en 60 secondes et $90 \%$ en une heure. Même si les entérotoxines ne sont pas excrétées, les reins constitueraient les principaux sites de dégradation. Les entérotoxines sont également absorbées par la peau. Le contact avec les entérotoxines peut produire des éruptions cutanées avec formation de vésicules suivies, sept jours plus tard, de desquamations des paumes [4]. Quelques heures après l'ingestion d'aliments contaminés, apparaissent les symptômes classiques de l'empoisonnement alimentaire : nausćes, vomissements, douleurs abdominales, diarrhée, maux de tête et crampes. Contrairement aux entérotoxines produites par d'autres bactéries, leur action ne se limite pas au tractus intestinal. A des concentrations beaucoup plus faibles que celles ayant lieu par la voie gastrique, l'administration intraveineuse de ces toxines à des animaux provoque vomissements et diarrhées. Seuls les humains et les singes sont sensibles aux entérotoxines, on note toutefois que les souris préalablement sensibilisées au Dgalactosamine (composé qui augmente leur susceptibilité aux médiateurs endogènes comme le TNF) subissent un choc létal après une injection intraveineuse de SEB [6].

On a commencé à s'intéresser vraiment aux propriétés immunologiques des entérotoxines qu'après avoir découvert leur activité mitogénique sur les lymphocytes. Les premières études ont montré que des lymphocytes périphériques humains et des cellules de rate de souris se multipliaient en présence de SEB [7]. En réalité, ce sont les lymphocytes $T$, aussi bien cytotoxiques (Tc) qu'auxiliaires (Th), qui sont activés par ces superantigènes. La prolifération des cellules $\mathrm{T}$ 
Tableau I

PROPRIÉTÉS DES SUPERANTIGĖNES

\begin{tabular}{|c|c|c|c|c|c|c|c|c|}
\hline TYPE & $\begin{array}{l}\text { PM } \\
\text { (Da) }\end{array}$ & $\begin{array}{l}\text { Acides } \\
\text { Aminés }\end{array}$ & pl & $\begin{array}{l}\text { PONT' } \\
\text { S-S }\end{array}$ & & $\begin{array}{l}\text { Kde } \\
\text { (M) }\end{array}$ & $v \beta$ murines $c$ & $v \beta$ humaines $c$ \\
\hline SEA & 27,078 & 233 & 7.3 & OUI & $8.2 \times$ & $\times 10-8$ & $1,3,11$ & $1.1,5 \cdot 3,6 \cdot 3-\cdot 5,7.4,9 \cdot 1,21.1^{d}$ \\
\hline SEB & 28,366 & 239 & 8.6 & OUI & $2.4 \times$ & $\times 10^{-7}$ & $3,7,8 \cdot 1-.3,11,17$ & $3,12,14,15,17,20$ \\
\hline SEC 1 & 27,496 & 239 & 8.6 & OUI & $7.4 \times$ & $\times 10^{-7}$ & $3,7,8.2-.3,11.17$ & 12 \\
\hline SED & 26,360 & 228 & 7.4 & OUI & $1.0 \times$ & $\times 10^{-6}$ & $3,7,8 \cdot 1-.3,11,17$ & 5,12 \\
\hline SEE & 26,425 & 230 & 7.0 & OUI & $1.1 \times$ & $\times 10^{-5}$ & $11,15,17$ & $5.1,6.1-.3,8,18$ \\
\hline CPE & 31,258 & 283 & 5.7 & NON & & & & \\
\hline ETB & 27,318 & 246 & 7.0 & NON & & & $1,3,8.2,10,11,15,17$ & 2 \\
\hline MAM & 27,000 & & $>9.0$ & & & & $6,8 \cdot 1-.3$ & 17 \\
\hline PE & 66,583 & 613 & 5.9 & & & & $3,5.1$ & $?$ \\
\hline SPEA & 25,787 & 221 & 5.0 & & & & & \\
\hline SPEB & 40,287 & 371 & 8.7 & NON & & & & \\
\hline SPEC & 24,354 & 208 & 6.7 & NON & & & & \\
\hline TSST-1 & 22,049 & 194 & 7.2 & NON & $4.4 \times$ & $\times 10^{-7}$ & $3,4,15,17$ & 2 \\
\hline VEA & $>10,000$ & & & & & & & \\
\hline
\end{tabular}

a. SEA à SEE, S. aureus entérotoxines A à E; CPE, Clostridium perfringens entérotoxine; ETB, S. aureus toxine exfoliative B ; MAM Mycoplasma arthritidis mitogène: PE, Pseudomonas aeruginosa exotoxine A; SPEA, SPEB, SPEC, Streptococcus pyogenes toxines A, B, C ; TSST-1, S. aureus toxine du syndrome du choc toxique: YEA, Yersinia entérocolitica antigène.

b. Pont disulfure.

c. compilees d'après [2,35].

d. John Fraser, communication personnelle.

e. Constante d'affinité avec HLA-DR1.

est cependant liée à la présence de cellules accessoires comme les monocytes ou les cellules B qui expriment à leur surface les molécules de classe II du complexe majeur d'histocompatibilité (CMH) [8]. Ces molécules agissent comme récepteurs pour les superantigènes (voir plus bas). Le mécanisme par lequel les entérotoxines et les autres superantigènes provoquent des pathologies sévères n'est pas encore clarifié. Leur activité résulte d'un effet synergique aux niveaux gastro-intestinal, métabolique et immunologique.

Les effets toxiques qui se manifestent peuvent avoir pour cause la fixation des superantigènes aux cellules intestinales par le truchement des récepteurs (peut-être les antigènes de classe II ou des molécules similaires), $m / s n^{\circ} 5$ vol. 9 , mai 93 suivie du relargage de neuropeptides. Ces molécules provoqueraient à leur tour la sécrétion, par l'intermédiaire des mastocytes, de leucotriènes et d'histamines, cause (parmi d'autres) de vomissements et de réactions cutanées [3]. L'interaction des entérotoxines avec différents types cellulaires exprimant à leur surface des molécules de classe II, provoque la synthèse de molécules potentiellement toxiques comme la cachectine (TNF) et l'interleukine-1 [9]. L'intoxication par les entérotoxines modifie aussi les taux plasmatiques de plusieurs métabolites susceptibles de faire surgir des symptômes associés à l'intoxication [10].

Lors de l'administration intraveineuse, la toxicité est aussi causée par l'action mitogénique déclenchée sur les lymphocytes $\mathrm{T}$ et la production de lymphokines. L'administration de SEB à des souris entraîne une perte de poids significative ainsi qu'une immunosuppression. Le rôle des cellules $\mathrm{T}$ dans ce phénomène a été mis en évidence par le groupe de Kappler et Marrack : 1) des souris $\mathrm{nu}^{+} / \mathrm{nu}^{+}$ (donc presque entièrement dépourvues de cellules $T$ ) ou 2) des souris dépourvues de cellules capables de réagir contre $\mathrm{SEB}$ ou 3 ) des souris immunosupprimées avec la cyclosporine $\mathrm{A}$, ne perdent pas de poids à la suite d'injections intrapéritonéales avec SEB [2]. L'utilisation de SEB recombinante fournit une preuve supplémentaire du rôle des cellules $\mathrm{T}$ dans la toxicité des entérotoxines. En effet cette molécule, rendue incapable d'interagir avec le RcT mais se liant 
toujours aux molécules de classe II, ne provoque pas de perte de poids [11]. Paradoxalement les cellules $\mathrm{T} \mathrm{CD}^{+}$activées par les superantigènes deviendront par la suite anergiques (paralysie fonctionnelle) ou seront éliminées par apoptose (mort cellulaire programmée) (figure 1) [12]. La nécessité d'une interaction entre les entérotoxines et le RcT demeure cependant à démontrer pour que se déclarent les symptômes de l'empoisonnement alimentaire et ceux des autres maladies causées par les staphylocoques.

\section{RÉFÉRENCES}

11. Kappler JW, Herman A, Clements J, Marrack P. Mutations defining functional regions of the superantigen staphylococcal enterotoxin B. J Exp Med 1992; 175 : 387-96.

12. Kawabe Y, Ochi A. Programmed cell death and extrathymic reduction of $\mathrm{V} \beta 8$ CD4 $\mathrm{T}$ cells in mice tolerant to Staphylococcus aureus enterotoxin B. Nature 1991; 349 : 245-8.

13. Fraser JD). High affinity binding of staphylococcal enterotoxins A and B to HI.A-DR. Nature $1989 ; 339$ : 221-3.

14. Chintagumpala MM, Mollick JA, Rich RR. Staphylococcal toxins bind to different sites on HLA-DR. J Immunol 1991; 147 : 3876-81.

15. Dellabona P, Peccoud J, Kappler J, Marrack P, Benoist C, Mathis D. Superantigens interact with $\mathrm{MHC}$ class II molecules outside of the antigen groove. Cell 1990 ; 62 : 1115-21.

16. Herman A, Labrecque $\mathrm{N}$, Thibodeau J, Marrack P, Kappler JW, Sekaly RP. Identification of the staphylococcal enterotoxin A superantigen binding site in the $\beta 1$ domain of the human histocompatibility antigen HLA-DR. Proc Natl Acad Sci USA 1991 ; 88: 9954-8.

17. Fraser JD, Urban RG, Strominger JL, Robinson $\mathrm{H}$. Zinc regulates the function of two superantigens. Proc Natl Acad Sci USA $1992 ; 89: 5507-11$.

18. Jorgensen JL, Reay PA, Ehrich EW, Davis MM. Molecular components of T-cell recognition. Ann Rev Immunol 1992; 10 : 835-73.

19. Panina-Bordignon $\mathrm{P}, \mathrm{Fu} \mathrm{X}$, Lanzavecchia A, Karr RW. Identification of HLADR alpha chain residues critical for binding of the toxic shock syndrome toxin superan-

\section{Les molécules de classe II du CMH sont les récepteurs des toxines bactériennes}

Les molécules de classe II du $\mathrm{CMH}$ constituent le principal récepteur cellulaire des superantigènes bactériens. Elles sont exprimées de façon constitutive par les lymphocytes B, les cellules dendritiques, les macrophages et les cellules épithéliales du thymus et de l'épithélium intestinal. Élément de réponse immunitaire à un microbe, la fonction de ces molécules consiste à présenter des peptides antigéniques aux lymphocytes. Ce sont des molécules hétérodimériques dont chacune des chaînes $\alpha$ et $\beta$ est composée de deux portions extracellulaires similaires aux immunoglobulines. Le peptide antigénique est fixé dans une cavité formée par des hélices $\alpha$ et des feuillets $\beta$ de chacune des chaînes. C'est dans cette région que sont locapolymorphiques. Plusieurs équipes de recherche ont réussi à immunoprécipiter des molécules de classe II en utilisant des toxines comme SEA ou $\mathrm{SEB}$, elles ont ainsi démontré clairement que les molécules de classe II étaient les récepteurs cellulaires des superantigènes bactériens [8]. Ceuxci, contrairement aux peptides antipour se lier aux molécules de classe II [7]. Des expériences de liaison ont démontré que les superantigènes bactériens manifestent une haute affinité avec les molécules de classe II. Le Tableau I indique que cette affinité varie entre $10^{-5}$ et $10^{-8} \mathrm{M}$ pour les différentes toxines. De plus, la stoechiométrie de cette lisés la majorité des acides aminés géniques, n'ont pas à être dégradés interaction indique un rapport de un pour un soit une molécule de classe II pour une molécule de toxine donnée. Les différents sérotypes de toxines de Staphylococcus ont pourtant des sites de contact distincts sur les molécules de classe II. Ainsi la liaison de SEA abolit la fixation subséquente de SEB et de TSST; par contre, SEB et TSST-1 auraient des sites de liaison distincts puisqu'elles peuvent se lier simultanément au même récepteur $[13,14]$.

Des études exhaustives ont révélé plus tard que toutes les molécules de classe II n'interagissaient pas de façon identique avec les toxines. Chez la souris, l'allèle $I-A^{k}$ ne présente qu'une très faible affinité avec TSST alors que $\mathrm{I}-\mathrm{A}^{\mathrm{b}}$ lie cette même toxine de façon très efficace. Chez l'humain, la fixation de TSST-1 par l'isotype HLA-DP est à peine détectable alors que les allèles de HLA-DR montrent une haute affinité avec TSST-1. Des observations similaires ont été rapportées pour SEA : l'allèle HLA-DR 1 affiche une haute affinité avec SEA alors qu'un autre allèle, HLADRw53, ne peut fixer cette toxine. Ces différences ont été attribuées au polymorphisme allélique de la chaîne $\beta$ : tous les allèles de DR s'associent à une chaîne $\alpha$ identique. Une mutagenèse exhaustive des résidus polymorphiques localisés sur la chaîne $\alpha$ de I-A - impliqués dans la présentation des peptides antigéniques aux cellules $\mathrm{T}-$ n'affecte pas la présentation des toxines (SEB, SEC) aux lymphocytes $\mathrm{T}[15]$. Ces travaux indiquent que le site de liaison des toxines est localisé à l'extérieur de la niche à peptides. En conformité avec ces résultats, il a été démontré que l'histidine en position 81 de la chaîne $\beta$ de HLA-DR est essentielle à la liaison de SEA (figure 2) [16]. Elle jouerait un rôle dans la formation d'un lien métallique $\mathrm{Zn}^{++}$avec des résidus histidine sur les toxines [17]. D'autres résidus voisins de l'histidine 81 sur l'hélice $\alpha$ de la chaîne $\beta$ sont aussi associés à la liaison de SEA aux molécules de classe II [18]. Quant à TSST-1, la chaîne $\alpha$ aurait un rôle prépondérant dans la liaison de cette toxine. En effet, la substitution de la chaîne $\alpha$ de HLADR (qui lie TSST-1 efficacement) par celle correspondante de I-E (qui pos- 


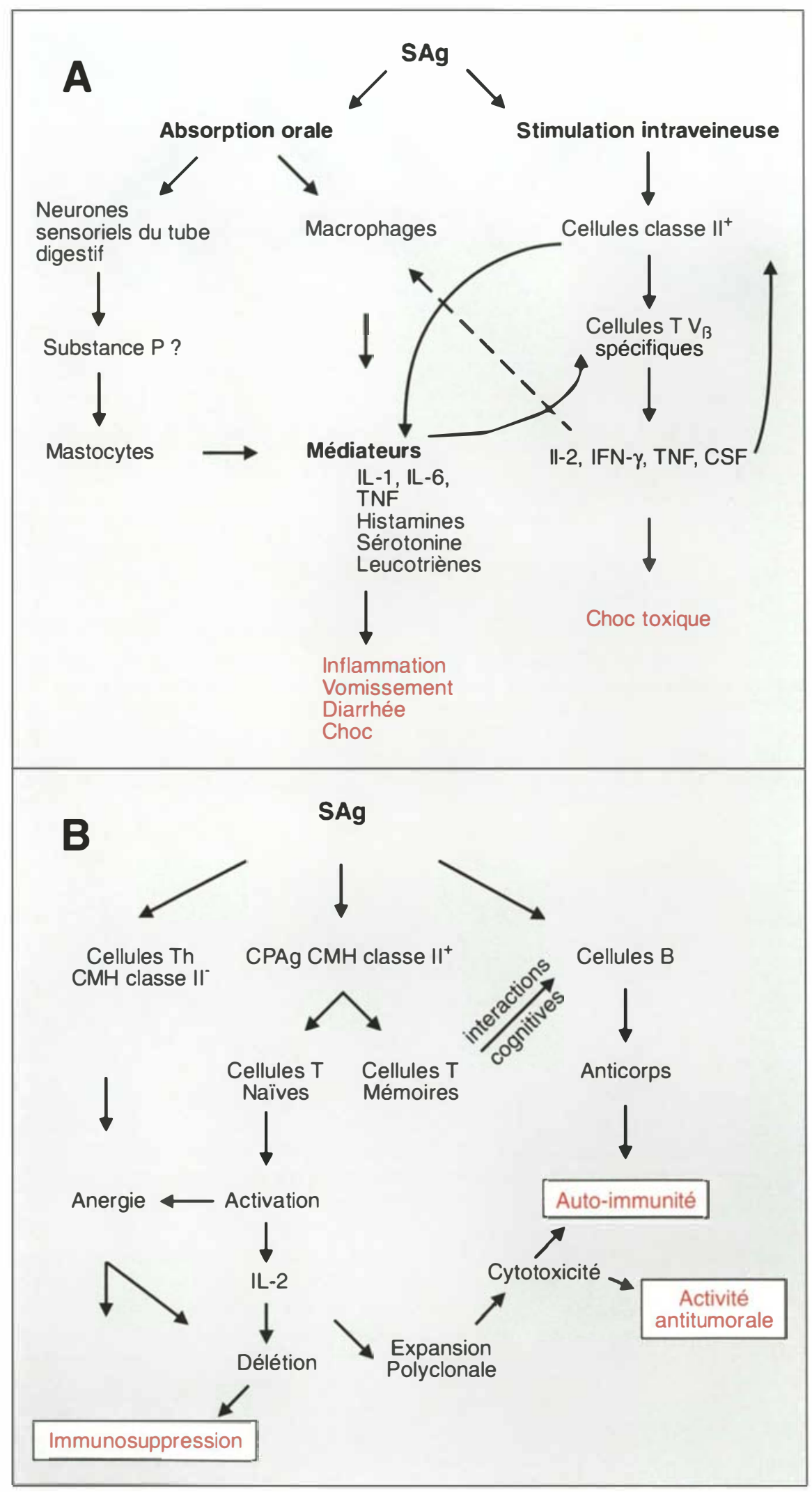

Figure 1. Propriétés biologiques des superantigènes. A) Cellules et cytokines participant à la toxicité des entérotoxines. B) Conséquences pathologiques et thérapeutiques possibles à la suite de l'absorption de superantigènes. $\mathrm{m} / \mathrm{s} n^{\circ} 5$ ool. 9, mai 93 sède une faible affinité pour TSST-1) abolit la liaison de TSST-1 à HLADR. L'utilisation de molécules chimériques entre différents allèles de souris ou différents allèles humains laisse penser que TSST-1 interagit avec la région hypervariable 2 de la chaîne $\alpha$. Des résultats récents de mutagenèse ont confirmé que les acides aminés critiques pour la fixation de cette toxine, sont localisés sur la chaîne $\alpha$ et en dehors de la niche à peptide [19]. Cette conclusion n'exclut pas un contact simultané avec la chaîne $\beta$. Tout en sachant que certains allèles de classe II ne lient pas les toxines, on ne peut imaginer qu'un individu soit résistant car une cellule exprime plusieurs molécules de classe II différents.

\section{Interactions avec le RcT}

Les superantigènes bactériens stimulent les lymphocytes $T$ exprimant des familles distinctes de régions variables de la chaîne $\beta$ du $\operatorname{RcT}[2,18,20]$. La spécificité de chacune des toxines de $S$. aureus pour les différentes régions variables du RcT est résumée au Tableau $I$. Les régions variables interagissant avec une toxine donnée, partagent une séquence commune localisée entre les résidus 68 à 74 . Des échanges réciproques de cette boucle entre des régions variables de chaîne $\beta$ ayant des spécificités pour des toxines différentes ont confirmé le rôle que ces acides aminés jouent dans la reconnaissance des superantigènes. Cette boucle, appelée CDR 4 (Complementarity Determining Region), présente une grande variabilité de sa séquence d'acides aminés; elle est située en retrait par rapport aux sections de la chaîne $\beta$ du RcT engagées dans la reconnaissance des peptides antigéniques. Cette constatation confirme que la topologie de l'interaction entre les toxines et les cellules $T$ est différente de la reconnaissance antigénique. Le rôle de la chaîne $\alpha$ du RcT dans la reconnaissance des superantigènes bactériens n'est pas bien établi. Ainsi, des chaînes $\beta$ solubles du RcT peuvent se lier à des complexes classe II-superantigènes bactériens en l'absence de chaîne $\alpha$ [21]. Par ailleurs, des lymphocytes $\mathrm{T}$ exprimant une région $\mathrm{V} \beta$ spécifique ne 
répondent pas toujours à la toxine laissant supposer ainsi que dans certains cas, la chaîne $\alpha$ pourrait influencer la formation du complexe trimoléculaire RcT-superantigèneclasse II.

Le rôle des différentes molécules de ce complexc est d'ailleurs encore discuté et fait l'objet de plusieurs recherches. La nécessité d'une interaction entre les molécules de classe II et le RcT est encore débattue (figure 3). Deux observations permettent de supposer qu'il n'existe pas d'interaction entre les molécules de classe II et le RcT lors de la présentation des superantigènes. Tout d'abord les toxines sont reconnues par les lymphocytes $\mathrm{CD}^{+}$qui sont restreints par les molécules classe II, mais aussi par ceux $\mathrm{CD}^{+}$ayant une spécificité antigénique limitée par la présence des molécules classe I du CMH. De plus, les lymphocytes $\mathrm{T}$ reconnaissent ces toxines liés à plusieurs allèles des molécules classe II du $\mathrm{CMH}[7,8$, 22]. D'autres résultats laissent cependant croire que le RcT interagit, non seulement avec la toxine, mais aussi simultanément avec la molécule de classe II. Une analyse plus fine, utilisant une séric de lymphocytes $\mathrm{T}$

\section{RÉFÉRENCES}

20. Herman A, Kappler JW, Marrack P, Pullen AM. Superantigens : mechanism of $\mathrm{T}$-cell stimulation and role in immune responses. Ann Rev Immunol 1991 ; 9 : 745-72.

21. Gascoigne NRJ, Ames KT. Direct binding of secreted $T$-ccll receptor $b$ chain to superantigen associatcd with class II major histocompatibility complex protein. Proc Natl Acad Sci USA 1991; 88 : 613-6.

22. Herrmann T, Maryanski JL, Romero P, Fleischer B, MacDonald HR. Activation of MHC class I-restricted CD8 CTL by microbial $\mathrm{T}$ ccll mitogens. Dependence upon MHC class II expression of the target cells and $\mathrm{V}$ usage of the responder $\mathrm{T}$ cells. $J$ Immunol 1990 ; 144: 1181-6.

23. Stelma GN, Bergdoll MS. Inactivation of staphylococcal enterotoxin A by chemical modification. Biochem Biophys Res Comm $1982 ; 105: 121-6$

24. Grossman D, Van M, Mollick JA, Highlander SK, Rich RR. Mutation of the disulphide loop in staphylococcal enterotoxin A. Consequences for $\mathrm{T}$ ccll recognition. $J$

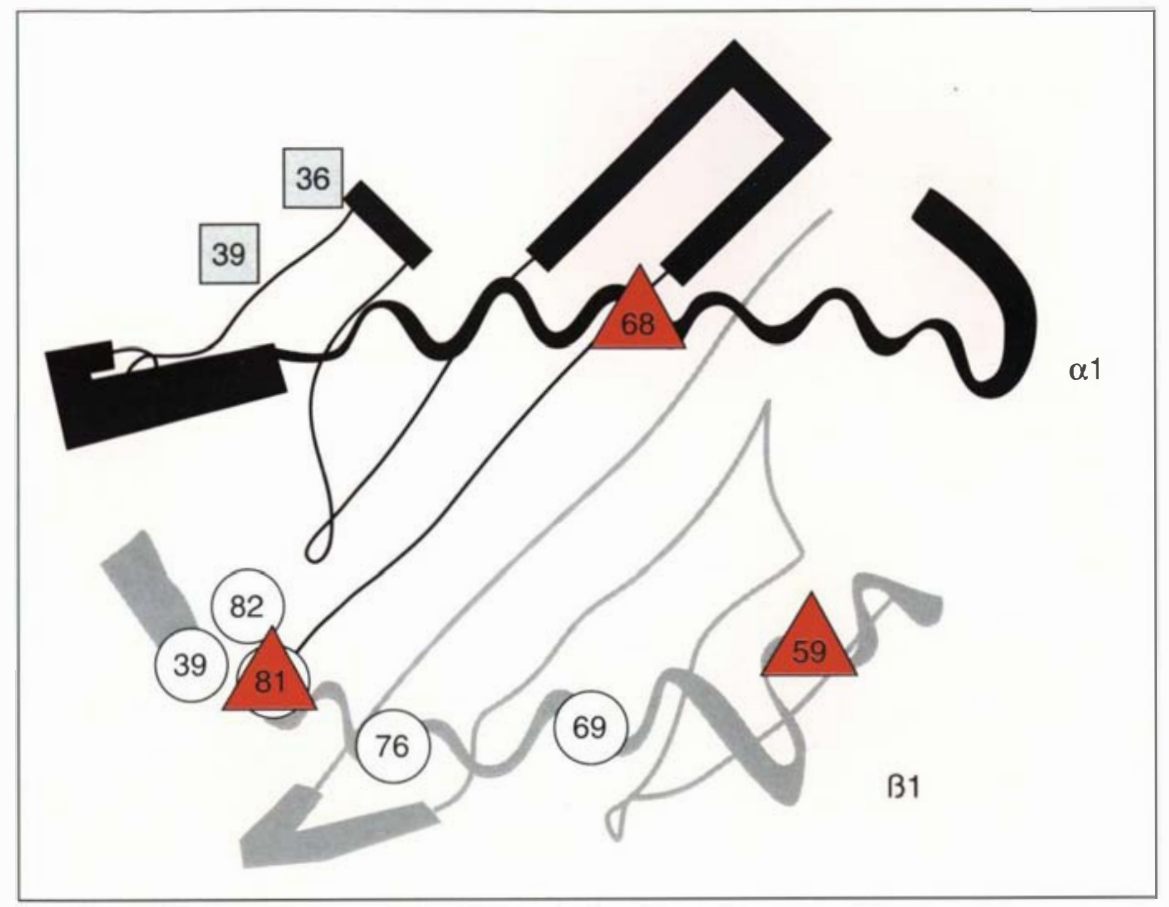

Figure 2. Sites de contact des entérotoxines et du Rct sur les molécules de classe II. Ce schéma représente une vue de haut lce que verrait le RcT) des domaines $\alpha 1$ et $\beta 1$ de la molécule HLA-DR1. Les cercles et les carrés indiquent les acides aminés ayant un rôle dans l'interaction avec SEA et TSST-1 respectivement. Les triangles indiquent les résidus critiques pour la présentation de SEA et/ou TSST-1 et donc en jeu dans le contact du RcT.

exprimant des RcT différents, indique que l'efficacité de la réponse varie sclon l'allèle utilisé pour la présentation des toxines. De plus, des molécules de classe II mutées sur des résidus mobilisés dans des interactions avec le RcT, ne présentent plus la toxine à certains lymphocytes T $\mathrm{mal}$ gré une liaison efficace des toxines (Labrecque N., Thibodeau J., Sékaly R.P., manuscrit en préparation) (figure 2). Une telle interaction du RcT avec les molécules du $\mathrm{CMH}$ pourrait s'établir par l'intermédiaire de la chaîne $\alpha$ du RcT.

\section{Domaines actifs des entérotoxines}

Les recherches sur la structure des entérotoxines se sont attardées à identifier les régions responsables 1) des propriétés émétiques, 2) de la liaison aux molécules de classe II et 3 ) du contact avec le RcT. Les premières expériences ont démontré qu'il était possible de séparer l'activité émétique des entérotoxines de l'activité mitogé- nique [23]. En ce qui concerne les sites de contact des molécules de classe II ou du RcT, les résultats de ces expériences ont démontré que ces régions étaient entremêlées [11]. La structure tridimensionnelle du cristal SEB récemment établie a permis de mieux définir ces 2 différents domaines fonctionnels (figure 4) [5]. Le site d'interaction avec le RcT se présente sous la forme d'une cavité formée par des résidus situés dans les portions $\mathrm{N}$ et C-terminale de SEB. Le site de contact avec la molécule de classe II n'est pas aussi bien défini. Les résidus identifiés lors des études de mutagenèse sont tous situés sur la même face de l'entérotoxine et témoignent de plusieurs points de contact avec les molécules de classe II. Les régions concernées sont cependant adjacentes ; un contact direct et spécifique est probablement possible entre les molécules de classe II et le RcT dans le complexe trimoléculaire. Une autre cavité se trouve sur le côté opposé au site de contact avec les molécules de classe II ; cette observa- 


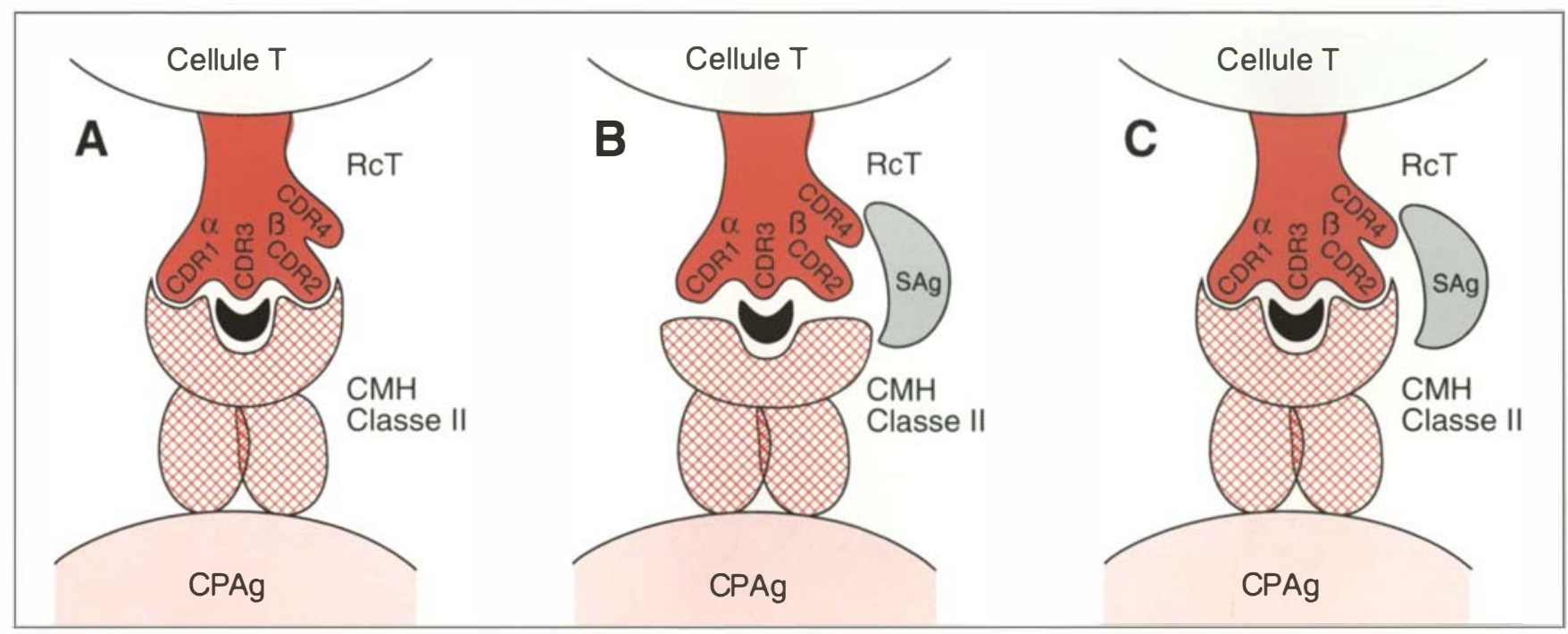

Figure 3. Modèles de la reconnaissance des superantigènes par les lymphocytes $\mathbf{T}$. A) Reconnaissance des antigènes conventionnels où le RcT interagit avec les molécules de classe II et le peptide. B) Les superantigènes sont reconnus indépendamment d'une interaction RcT-molécule de classe II. C) Les 3 molécules du complexe et probablement le peptide interagissent les unes avec les autres.

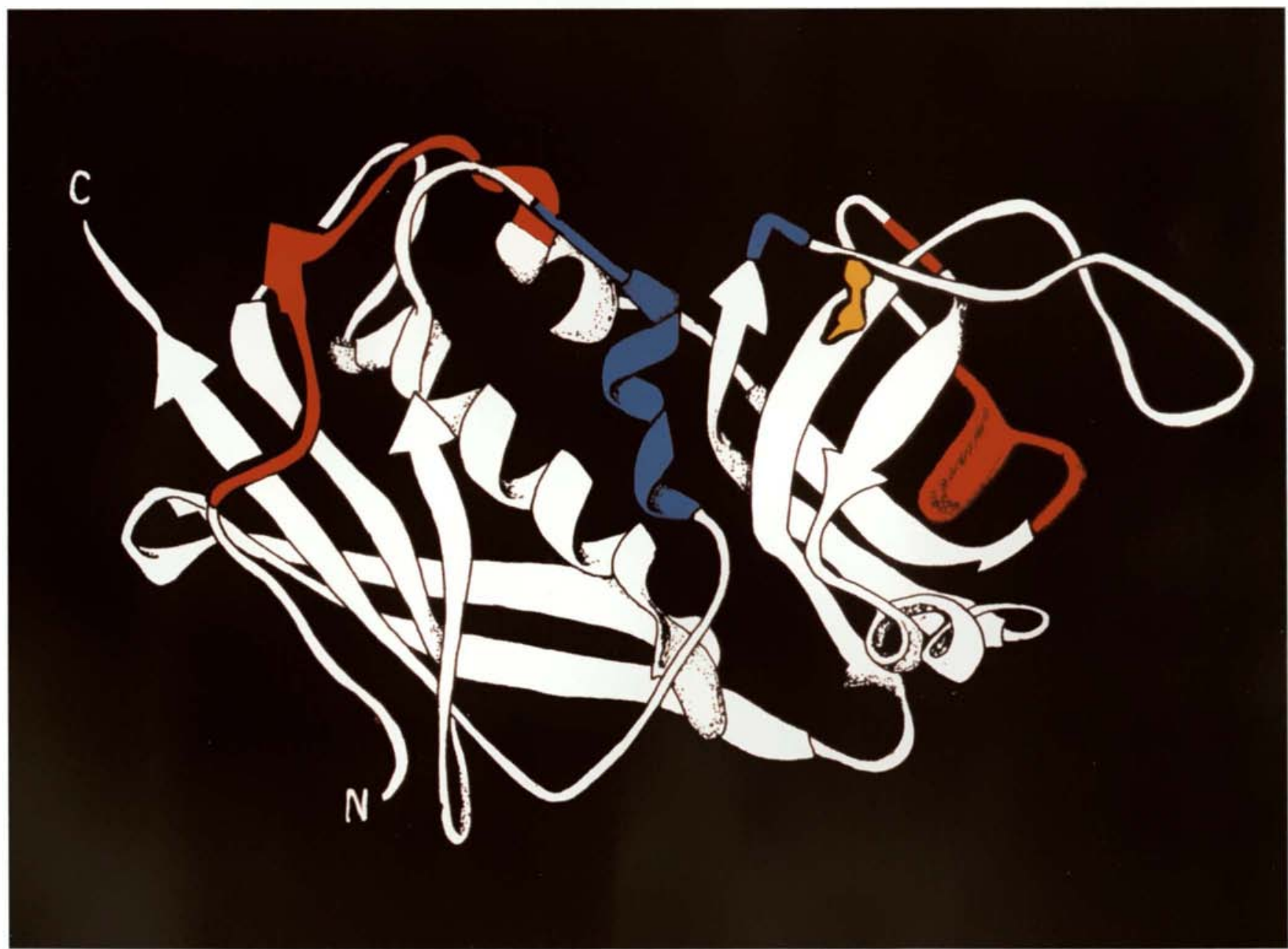

Figure 4. Représentation schématique de la structure de SEB. Les sites proposés de contact avec le RcT sont indiqués en rouge alors que les acides aminés entrant en jeu la fixation des molécules de classe II sont situés dans les portions bleues. Le pont disulfure est en jaune. Adapté d'après Swaminathan et al. [5]. $\mathrm{m} / \mathrm{s} n^{\circ} 5$ vol. 9 , mai 93 


\section{RÉFÉRENCES}

25. Friedman SM, Posnett DN, Tumang JR, Cole BC, Crow MK. A potential role for microbial superantigens in the pathogenesis of systemic autoimmune disease. Arthritis Rheum 1991 ; 34 : 468-79.

26. Wucherpfenning $\mathrm{KW}$, Ota $\mathrm{K}$, Endo $\mathrm{N}$, Seidman JG, Rosenzweig A, Weiner HL, Hafler DA. Shared human T cell receptor $\mathrm{V} \beta$ usage to immunodominant regions of myelin basic protein. Science 1990; 248 : 1016-9.

27. Kim C, Siminovitch KA, Ochi A. Reduction of lupus nephritis in MRL/lpr mice by a bacterial superantigen treatment. $J$ Exp Med 1991; 174：1431-7.

28. Dohlsten M, Hedlund G, Kalland T. Staphylococcal-enterotoxin-dependent cellmediated cytotoxicity. Immunol Today 1991 ; $12: 147-50$.

29. Dohlsten M, Hedlund G, Segren S, Lando PA, Herrmann T, Kelly AP, Kalland T. Class II-negative colon carcinoma cells present staphylococcal superantigens to cytotoxiclymphocytes-T : Evidence for a novel enterotoxin receptor. Eur J Immunol $1991 ; 21: 1229-33$.

30. Dohlsten M, Hedlund G, Akerblom E, Lando PA, Kalland T. Monoclonal antibody-targeted superantigens-A different class of anti-tumor agents. Proc Natl Acad Sci USA 1991; 88 : 9287-91.

31. Rosenberg SA, Lotze MT. Cancer immunotherapy using interleukin-2 and interleukin-2-activated lymphocytes. Ann Rev Immunol 1986 ; 4 : 681-709.

32. Wing MG, Montgomery AMP, Harley C, Lachman PJ. Cytostasis of different tumours by a murine PPD-reactive $\mathrm{CD} 4^{+}$ Tlymphocyte clone is mediated by interferon-gamma and tumour necrosis factor alone or synergistically. Clin Exp Immunol $1990 ; 82$ : 208-13.

33. Rott O, Wekerle H, Fleischer B. Protection from experimental allergic encephalomyelitis by application of a bacterial superantigen. Inf Immun 1992; 4 : 347-53.

34. Hewitt CRA, Lamb JR, Hayball J, Hill M, Owen MJ, O'Hehir RE. Major histocompatibility complex independent clonal $\mathrm{T}$-cell anergy by direct interaction of Staphylococcus aureus enterotoxin B with the $\mathrm{T}$ cell antigen receptor. $J$ Exp Med 1992 ; $175: 1493-9$.

35. Misfeldt ML. Microbial "Superantigens ". Inf Immun 1990 ; 58 : 2409-13.

36. Alber G, Hammer DK, Fleischer B. Relationship between enterotoxic- and $T$ lymphocyte-stimulating activity of Staphylococcal enterotoxin B. J Immunol 1990 ; 144 : tion amène à considérer qu'une autre molécule pourrait interagir simultanément avec le complexe trimoléculaire. Cette région comprend 3 des 5 histidines de SEB et serait utile dans la reconnaissance d'un autre récepteur, responsable, celui-là, des manifestations entériques par exemple. En effet, il a été démontré que la carboxyméthylation de ces résidus abolit l'activité émétique de SEA et SEB [23, 36].

La boucle, formée par le pont disulfure, est en contact avec le solvant et est située à proximité du site de liaison du récepteur $\mathrm{T}$. La destruction du pont disulfure affecte le pouvoir potentiel mitogénique, sans toutefois affecter la spécificité de l'interaction avec le récepteur $T$ [24]. La spécificité des différents superantigènes pour une $V \beta$ particulière semble imposée par d'autres acides aminés situés en bordure du site d'interaction avec le récepteur T. Confirmant les propriétés inhérentes à la structure cristalline, des expériences récentes ont démontré que la liaison entre SEA et une chaîne $\mathrm{V} \beta 3$ soluble peut être abolie au profit d'une chaîne $\mathrm{V} \beta 11$, en changeant seulement 3 acides aminés dans la région C-terminale de SEA [5].

Les travaux sur la structure du cristal nous aiderons maintenant à orienter la mutagenèse des entérotoxines afin de mieux définir les régions participant à l'interaction avec différents ligands. Soulignons ici que les entérotoxines présentent vraisemblablement une structure tertiaire similaire puisque les résidus variables sont situés dans les tours, dans les boucles ou dans les régions exposées au solvant.

\section{Entérotoxines et auto-immunité}

L'auto-immunité exprime la rupture de la tolérance accordée aux cellules du système immunitaire susceptibles de mobiliser les réactions contre le " soi ". Bien qu'aucune preuve directe ne relie les entérotoxines aux maladies auto-immunitaires, plusieurs faits évidents laissent penser maintenant que des superantigènes sont associés à leur développement [25]. Mycoplasma arthritidis représente un excellent modèle pour l'arthrite rhu- matoïde. Cette bactérie produit un facteur mitogénique soluble (MAM) dont les propriétés superantigéniques seraient reliées au développement de la maladie chez le rat. Dans d'autres maladies auto-immunitaires comme, par exemple, la sclérose en plaques chez l'humain et le lupus néphrotique chez la souris, on trouve chez les individus souffrant de ces maladies une augmentation de cellules $\mathrm{T}$ exprimant une $V \beta$ donnée $[26,27]$. Cette prédominance $V \beta$ n'est pas toujours observée, elle peut varier selon le stade de la maladie ; cependant, ce phénomène rappelle l'action des superantigènes sur le répertoire des cellules T. A la suite d'une infection, les superantigènes provoqueraient la maladie d'au moins trois façons. Premièrement, une auto-immunité spécifique d'organe pourrait résulter de l'activation de cellules Tc ou $\mathrm{Th}$ auto-réactives épargnées par l'inactivation. Le transfert de la maladie EAE par l'injection de cellules $\mathrm{CD}^{+}{ }^{+}$confirme l'importance de ces cellules. La lyse des cellules classe $\mathrm{II}^{+}$provoquée par les lymphocytes T ainsi activés (SDCC) affaiblirait le système immunitaire et laisserait la bactérie en tirer avantage [28]. Une deuxième possibilité serait que le superantigène se lie aux molécules de classe II sur les cellules $\mathrm{B}$; il permettrait ainsi une interaction cognitive avec les cellules $T$ arborant le segment $\mathrm{V} \beta$ complémentaire. Des interactions de ce genre, menant à l'activation des cellules $\mathrm{B}$ et à la production d'anticorps, ont été mises en évidence in vitro et in vivo en utilisant le superantigène MAM [25]. Troisièmement, l'activation partielle des cellules $B$ par les superantigènes circulants suffirait à établir un bassin de cellules B mémoires qui, en présence d'un antigène ou de lymphokines, produiraient des anticorps dont certains seraient spécifiques du soi.

\section{Potentiel} thérapeutique

L'utilisation des entérotoxines comme agents antitumoraux est actuellement envisagée. Plusieurs équipes ont déjà démontré in vitro la capacité de certaines entérotoxines de provoquer la lyse de cellules cancéreuses par des 
lymphocytes $\mathrm{T}$ cytotoxiques. On a obtenu une réduction significative de l'apparition de tumeurs à la suite d'un traitement avec SEB chez des souris $\mathrm{C} 3 \mathrm{H}$ inoculées avec la lignée tumorale 1691-PRO4L provoquant des cancers de la peau [29]. L'injection de cette toxine entraîne l'augmentation de lymphocytes T $\mathrm{CD}^{+}$ $\mathrm{V} \beta 8^{+}$exprimant le récepteur de l'IL-2 ; ces derniers seraient directement responsables de la régression des tumeurs. Mais comme l'injection de toxines in vivo pourrait avoir des effets désastreux sur l'organisme, il faut qu'elles soient bien concentrées dans le site même de la tumeur; c'est pourquoi les toxines pourraient être couplées chimiquement à des anticorps spécifiques des cellules transformées. De telles molécules (conjuguées anticorps-SEA) se sont avérées capables, par des lymphocytes $\mathrm{T}$ cytotoxiques, de produire la lyse de cellules classe $\mathrm{II}^{-}$de carcinome du colon [30]. Ce résultat est surprenant puisque en général la présentation de toxines en l'absence de molécules de classe II aboutit à une paralysie fonctionnelle des lymphocytes T. L'oligomérisation de complexes anticorps-toxines (" capping ") produirait dans ce modèle l'activation des lymphocytes $\mathrm{T}$ cytotoxiques. Plusieurs protocoles thérapeutiques consistant en une injection systémique de fortes concentrations d'IL-2 sont en cours ; malgré des débuts prometteurs, cette forme d'immunothérapie est en général mal tolérée par l'organisme [31]. L'injection de complexes anticorpstoxines éviterait ce problème. Ces complexes s'achemineraient directement au site de la tumeur et induiraient la production localisée d'IL-2. Celle-ci aurait pour effet de restaurer l'activité fonctionnelle des lymphocytes $\mathrm{T}$ qui s'infiltrent dans la tumeur et qui sont, en général, anergiques.

D'autres cytokines, comme l'interféron- $\gamma$ et la cachectine (TNF $\alpha$ et $\beta$ ), qui sont généralement sécrétées à la suite de l'activation des lymphocytes $\mathrm{T}$ par les toxines, pourraient faciliter la régression des tumeurs. La cachectine lyserait directement les cellules tumorales alors que l'interféron- $\gamma$ entraînerait la libération des molécules de classe II à leur surface. Cette libération aurait pour effet de recruter des lymphocy- tes $\mathrm{T} \mathrm{CD4}^{+}$(Tc ou Th) exclusifs à la cellule tumorale, augmentant ainsi la réponse cellulaire [32]. Il est probable que ces toxines se fixeraient sur toutes les cellules exprimant des molécules de classe II, ce qui aurait pour effet de les empêcher de se rendre dans le site de la tumeur. La liaison de ces conjugués avec lymphocytes $\mathrm{T}$ en l'absence de certains cosignaux spécifiques émis par des cellules spécialisées entraînerait l'anergie ou l'apoptose des lymphocytes T. Le site de liaison des entérotoxines avec des molécules de classe II devrait donc être préalablement éliminé par manipulations génétiques.

Les modèles de maladies autoimmunitaires indiquent que des lymphocytes $\mathrm{T}$, portant des RcT ayant un segment $V \beta$ particulier, joueraient un rôle dans le développement de ces maladies. L'utilisation des entérotoxines pour éliminer ces lymphocytes, par apoptose ou par anergie, a déjà été proposée. C'est ainsi qu'une réduction de lupus néphrotique a été observée chez la souris après un traitement avec des superantigènes bactériens [27]. D'autre part, des rats Lewis n'ont pas été atteints d'une encéphalomyélite allergique expérimentale à la suite de l'injection de SEE [33]. L'anergie serait donc provoquée par le contact du RcT avec le superantigène soluble, c'est-à-dire non lié aux molécules de classe II (figure 1) [34]. L'utilisation à nouveau d'entérotoxines mutées dans le site de liaison de molécules de classe II éviterait la production indésirable de lymphokines et d'anticorps.

\section{Conclusion}

Les expériences effectuées sur les entérotoxines de $S$. aureus constituent de bon moyens d'étudier les composantes structurales menant à l'activation des lymphocytes T. La compréhension de leur topologie rendra possible la mise au point de superantigènes spécifiques de chacune des molécules de classe II ou de chacun des récepteurs $T$. L'utilisation de molécules ne pouvant interagir avec le RcT nous aidera à concurrencer la fixation de toxines néfastes sur les molécules de classe II et ainsi à moduler la réponse immunitaire. Ces toxines synthétiques pourraient aussi empêcher le contact entre des cellules présentant des peptides antigéniques du soi et des lymphocytes $\mathrm{T}$ auto-réactifs. En effet plusieurs allèles des molécules de classe II sont reliés aux maladies autoimmunitaires ; on pourrait les rendre inefficaces sans même connaître la nature du peptide présenté. La découverte de superantigènes viraux comme ceux encodés par MMTV (mouse mammary tumor virus), dont la séquence primaire est très différente de leurs homologues bactériens, va permettre une analyse menant à des règles structurales dans le développement de superantigènes pharmacologiques [20]. Cependant le traitement de diverses pathologies grâce aux superantigènes demeure une perspective théorique. Plusieurs années de recherches seront nécessaires, aussi bien sur les superantigènes que sur les maladies auto-immunitaires, avant d'espérer sérieusement les utiliser à des fins thérapeutiques

\section{Remerciements}

J.T., N.L. et R.P.S. sont subventionnés par le Conseil de la Recherche Médicale du Canada. Les auteurs remercient V.V. Micusan pour plusieurs discussions stimulantes et François Denis pour son aide lors de l'analyse informatique.

\section{Summary}

Bacterial superantigens : pathogenic agents or therapeutic tools

Staphylococcus aureus enterotoxins are one of the major cause of food poisoning in man and these molecules are superantigens. They greatly affect the immune system and might be responsible for the development of autoimmune diseases. These toxins however could be used as therapeutics in many pathologies.

\section{TIRÉS A PART}

Rafick-Pierre Sékaly. 